\title{
Abordagem fisioterapêutica no tratamento da síndrome do estresse tibial medial - canelite em corredores de rua
}

Physiotherapeutic approach in the treatment of medial tibial stress syndrome - shin splints in street runners

Enfoque fisioterapéutico en el tratamiento del síndrome de estrés tibial medial - dolor en las espinillas en corredores callejeros

\section{Resumo}

Com o aumento exponencial de corredores de rua, houve também o aumento de lesões, dentre elas, a Síndrome do Estresse Tibial Medial (SETM), que é descrita como uma lesão na camada externa da tíbia, causando dor na região posteromedial, com tamanho de aproximadamente $\geq 5 \mathrm{~cm}$. O objetivo desse estudo é identificar os fatores de risco, como é realizado o diagnóstico e atuação fisioterapêutica para a SETM. Metodologia: trata-se de uma revisão de literatura, utilizando o método de pesquisa qualitativo, de caráter descritivo. Foi realizado um levantamento bibliográfico nos bancos de dados PubMed, Google Scholar e SciELO. Resultado: A causa exata da SETM é desconhecida, sendo mais aceito que se trata de uma condição multifatorial, fatores de risco identificados: o aumento do IMC; queda do navicular acima de 10mm; aumento da ADM de flexão plantar; plano de treinamento inapropriado; lesões previas no $2 / 3$ distal da perna e desequilíbrio de forças musculares. Seu diagnóstico é realizado através de avaliação clínica, contendo um exame histórico, seguido de um exame físico. Apesar da avaliação clínica ser padrão ouro, exames de imagens podem ser necessário em alguns casos específicos. Conclusão: Apesar da grande incidência da SETM não foi identificado durante a revisão da literatura deste estudo, um protocolo padrão considerado eficaz, portanto seu tratamento se concentra na apresentação clínica do paciente, sendo realizada intervenções generalizadas, carecendo assim de mais evidencias cientificas de boa qualidade para verificar a eficácia de determinada intervenção fisioterapêutica no tratamento especifico da SETM.

Palavras-chave: Síndrome do estresse tibial medial; Fatores de risco; Canelite.

\begin{abstract}
As an exponential increase in road corridors, there is also an increase in injuries, within them, to Medial Tibial Stress Syndrome (SETM), which is described as a lesion in the external layer of the tibia, causing the posteromedial region to have a size of approximately $\geq 5 \mathrm{~cm}$. The objective of this study is to identify the factors of the cliff, how it has been carried out or the diagnosis and physiotherapeutic treatment for the SETM. Methodology: it is about a literature review, using a qualitative research method, of a descriptive nature. A bibliographic survey was carried out in PubMed, Google Scholar and SciELO data banks. Result: Due to the exact cause of SETM is unknown, being more oily than it is a multifatorial condition, identified cliff factors: o increased BMI; navicular left above $10 \mathrm{~mm}$; increase in plantar flexion ADM; inappropriate treinamento plane; Previous injuries no 2/3 distal to the leg and imbalance of muscular forces. Its diagnosis was made through a clinical evaluation, containing a historical examination, followed by a physical examination. Despite the clinical evaluation being certified, image exams may be necessary in some specific cases. Conclusion: Despite the great incidence of SETM, it was not identified during the literature review of this study, a standard protocol considered effective, therefore its treatment is concentrated on the clinical presentation of the patient,
\end{abstract}


being generalized interventions, lacking further scientific evidence of good quality. to verify the effectiveness of a specific physiotherapeutic intervention, not specific treatment of SETM.

Keywords: Medial tibial stress syndrome; Risk factors; Shin splints.

\begin{abstract}
Resumen
Con el aumento exponencial de corredores callejeros, también hubo un aumento de lesiones, incluido el Síndrome de Estrés Tibial Medial (SETM), que se describe como una lesión en la capa externa de la tibia, que causa dolor en la región posteromedial, con tamaño de aproximadamente $\geq 5 \mathrm{~cm}$. El objetivo de este estudio es identificar los factores de riesgo, cómo se realiza el diagnóstico y el desempeño de la fisioterapia para SETM. Metodología: se trata de una revisión de la literatura, utilizando el método de investigación cualitativa, con carácter descriptivo. Se realizó un levantamiento bibliográfico en las bases de datos PubMed, Google Scholar y SciELO. Resultado: Se desconoce la causa exacta de SETM, siendo más aceptado que es una condición multifactorial, factores de riesgo identificados: aumento del IMC; caída del escafoides por encima de $10 \mathrm{~mm}$; aumento del ROM de flexión plantar; plan de entrenamiento inadecuado; lesiones previas en los 2/3 distales de la pierna y desequilibrio de fuerzas musculares. Su diagnóstico se realiza mediante evaluación clínica, que contiene un examen histórico, seguido de un examen físico. Aunque la evaluación clínica es el estándar de oro, los exámenes por imágenes pueden ser necesarios en algunos casos específicos. Conclusión: A pesar de la alta incidencia de SETM, no se identificó durante la revisión de la literatura de este estudio, un protocolo estándar considerado efectivo, por lo que su tratamiento se enfoca en la presentación clínica del paciente, realizándose intervenciones generalizadas, careciendo así de evidencia científica de mayor calidad. para verificar la efectividad de una determinada intervención fisioterapéutica en el tratamiento específico de SETM.
\end{abstract}

Palabras clave: Síndrome de estrés tibial medial; Factores de riesgo; Dolor en las espinillas.

\title{
1. Introdução
}

Recentemente a corrida de rua vem ganhando uma grande quantidade de adeptos, isso se explica por diversos fatores benéficos que a corrida de rua proporciona. De acordo com a pesquisa de Tohmatsu, (2011), durante aquele período a corrida de rua já se sagrava como o segundo esporte mais praticado no Brasil, divididos entre atletas amadores e profissionais.

Apesar dos diversos benefícios que a corrida de rua proporciona, o seu aumento exponencial também trouxe consigo o aumento do número de lesões. Essas lesões estão relacionadas a diversos aspectos intrínsecos e extrínsecos ao nosso corpo, ou seja, sendo resultante dos fatores interiores aqueles relacionados a individualidade biológica e os fatores exteriores, associadas as condições do meio ambiente que o corredor está inserido. (Fukuchi e Duarte, 2008).

A Síndrome do Estresse Medial Tibial (SETM) está intimamente relacionada a corrida de rua, pois essa modalidade pode aumentar consideravelmente a chance de o atleta adquirir uma inflamação na camada mais profunda da perna. Essa lesão tem a incidência maior em corredores de longas distâncias, pois sofrem um estresse acentuado de forma repetitiva em uma mesma região, isso porque a corrida gera em média forças de impacto que variam de 3 a 8 vezes maior do que a caminhada (Warden, Davis e Fredericson, 2014).

Quando falamos na correlação entre músculos e osso, é hipotetizado que o músculo é um tecido protetor fisiológico e não causador da SETM. Durante a fase de aterrissagem, o músculo atua como um dissipador de impacto, ajudando a diminuir as cargas que seriam levados aos ossos. Quando os músculos se encontram fatigados ou enfraquecidos, sua capacidade de dissipar as cargas ficam comprometidas, levando assim a um aumento de impacto ósseo. (Warden, Davis e Fredericson, 2014; Manoel, et.al. 2015).

De acordo com Yamato, et al. (2011), a grande maioria das lesões que acometem os corredores de rua não estão relacionado a nenhum evento especifico direto, como um trauma ou fratura, pelo contrário, o mais comum é que as lesões sejam provocadas por excesso de esforço em uma determinada estrutura. Os tipos de lesões nesse nicho podem variar, dependendo de alguns fatores próprio ao atleta, como sua raça, sexo, idade e massa corporal.

Entretanto, felizmente a maioria das lesões por esforço excessivo durante as corridas não resultam no afastamento do atleta das ruas, pois segundo Yamato, et al. (2011) nem sempre os atletas estão cientes dos sintomas de uma lesão por esforço excessivo, sendo inclusive, possível que o corredor continue praticando a corrida em fases iniciais da lesão. 
Isto posto, é notório que a corrida de rua, apesar de benéfica, propicia o surgimento de lesões em seus adeptos, portanto o objetivo desse estudo é fornecer informações a respeito dos fatores de risco, diagnostico e do tratamento fisioterapêutico na síndrome do estresse tibial medial em atletas corredores, melhorando assim, seu desempenho e qualidade de vida.

\section{Metodologia}

O presente estudo trata-se de uma revisão de literatura, tendo como base o método de pesquisa qualitativo, de caráter descritivo, afim de reunir um conjunto razoável de informações compatíveis com o tema. (Mazucato, 2018).

Para a seleção da literatura foi realizado um levantamento bibliográfico entre os meses de março e junho de 2021 nos bancos de dados Pubmed, Google scholar e sciELO, utilizando os seguintes descritores em português e inglês: "síndrome do estresse tibial medial (SETM) / medial tibial stress syndrome (MTSS)", "corrida de rua / street running", "abordagem fisioterapêutica / physiotherapeutic approach". Os critérios de inclusão e exclusão foi realizada por meio da leitura do título e resumo, caso se mostrassem compatíveis com o tema, seria realizado a leitura do texto completo do artigo. foram excluídos os artigos que não tinham clareza em sua fundamentação cientifica e/ou os que não estavam associados com o tema apresentado. Fazendo uso dos descritores citados, foram incluídos na revisão duas monografias e 27 artigos científicos compatíveis.

\section{Resultados e Discussão}

\subsection{Visão geral da síndrome do estresse medial tibial}

A síndrome de estresse tibial medial (SETM) é caracterizada por uma lesão ocasionada por uso excessivo das estruturas da região distal da perna. Esse tipo de lesão ocorre basicamente quando há um desequilíbrio entre a quantidade de micro lesões sofridas e a capacidade de cura que o osso possui. esse fenômeno é conhecido como lesão por uso excessivo ou overuse (Yamasaki, 2019; Viana, 2020).

Essa condição está diretamente ligada a musculatura do $2 / 3$ distal da perna. Dentre outros músculos dessa região, devemos dar uma devida atenção ao o tibial anterior, pois ele é considerado o principal executor da dorsiflexão e inversão de pé. Além desses movimentos isolados, o tibial anterior é bastante utilizado na corrida, realizando o movimento de elevação dos dedos a cada passo dado. Quando essa musculatura e tendão se inflamam por excesso de uso ou impacto, causam dor na face anterior da perna. (WalkeR, 2011).

Quanto a fisiopatologia da SETM Galbraith e Lavallee, (2009) diz que sua principal causa envolve a camada mais externa da tíbia, devido à tensão tibial sofrida durante a corrida. Entretanto, há estudos que indicam que há um espectro de outros fatores que influenciam no surgimento da SETM, dentre eles, podemos citar tendinopatias, periostite, déficit da capacidade de remodelamento periosteal e repetição de estresse tibial. A disfunção do tibial anterior, tibial posterior e sóleo, também são comumente aplicadas como predisponentes a SETM. Embora haja diversas etiologias, há o consenso de que a os principais sintomas gerados ao paciente é devido ao estresse ósseo. (Viana, 2020).

Segundo Yamasaki, (2019), geralmente a queixa mais comum dos pacientes portadores da SETM é de dor vaga e difusa em região medial distal da tíbia, geralmente com tamanho de cerca de $5 \mathrm{~cm}$. Nas fases iniciais da lesão, a dor é maior no início da corrida e vai diminuindo durante o treinamento. Conforme a lesão progride, a dor pode se apresentar até mesmo em descanso.

\subsection{Fatores de risco}

O plano de treinamento é algo que deve ser levado em consideração, podendo este influenciar de forma direta no surgimento da SETM. Um histórico abrangente deve ser obtido para avaliar a rotina de exercícios semanais do atleta e a quilometragem em execução, bem como a intensidade, ritmo, terreno e calçado. Erros no programa de treinamento geralmente é um dos fatores mais comuns envolvidos no surgimento da SETM. Alguns erros comuns de treinamento são o aumento 
exacerbado da intensidade ou duração logo no início recente da pratica da atividade física (Winters, et al., 2013). Também podemos citar a execução do treino em superfícies duras ou irregulares e indivíduos com lesões anteriores de membros inferiores, incluindo as demais lesões por estresse excessivo (Yamasaki, 2019).

Índice de massa corporal está diretamente associado ao surgimento da SETM. Isso acontece pois com o aumento do IMC, há também o aumento de carga sobre a estrutura óssea da tíbia, causando micro-danos no periósteo. Uma outra explicação está relacionada com a adaptação da tíbia aos exercícios. O osso é um tecido adaptável, porém, aparentemente a adaptabilidade se torna mais lenta em pacientes com o IMC aumentado, precisando de mais tempo para se adaptar as cargas sofridas durante a corrida. (Yates e White, 2004)

Teste de queda do navicular ou (Navicular drop test) geralmente é utilizado para mensurar a altura do arco plantar e da pronação do pé. O teste é considerado positivo quando houver diferença significativa do deslocamento do navicular durante uma descarga de peso, maior que 10mm, causando assim uma hiperpronação [Figura 1] (Sabino, et al., 2012)

Figura 1. Navicular drop test sendo realizado, evidenciando uma queda do arco plantar exacerbado, caracterizando um teste positivo

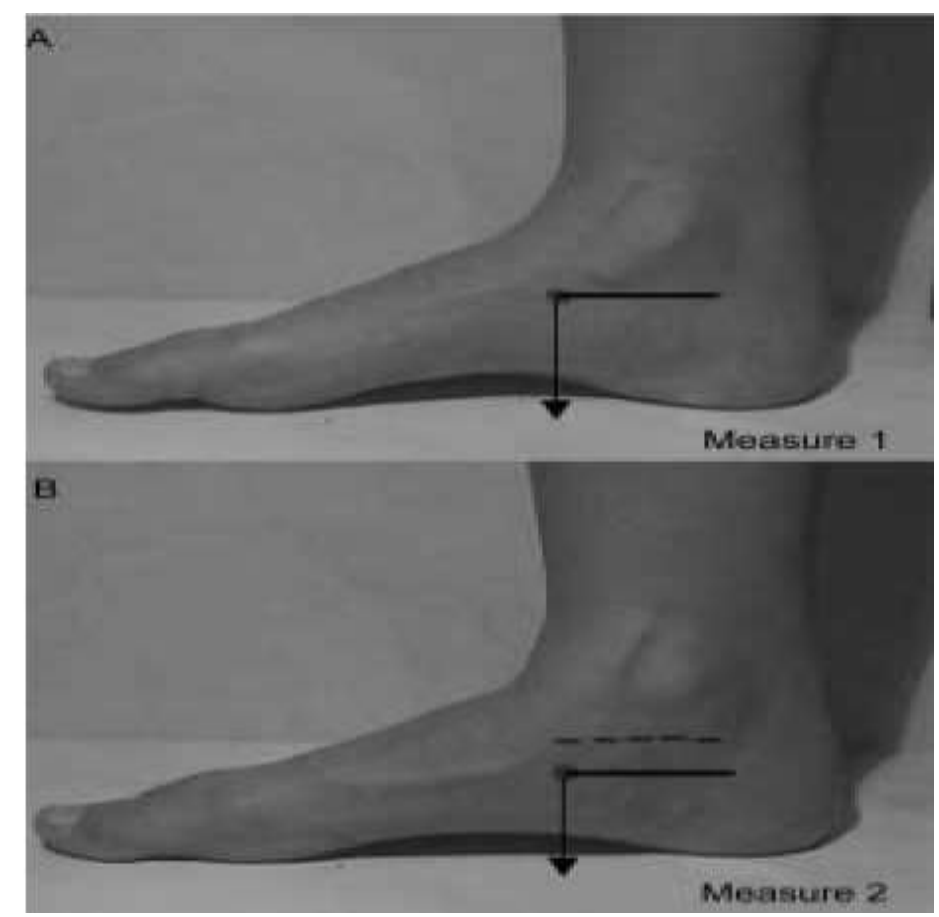

Fonte: https://www.jospt.org/doi/10.2519/jospt.2004.34.4.201

A pronação do pé é considerada um movimento natural do corpo durante a corrida como forma de proteção, pois permite que as cargas sejam dissipadas de forma mais hemogenia. A queda do navicular e a altura do arco estão diretamente relacionados à rotação medial da tíbia. Uma possível explicação é que a diminuição da rotação medial da tíbia, interfere diretamente na diminuição de absorção de impacto promovido pela pronação de pé, provocando assim uma maior sobrecarga na própria tíbia. (YagI, Muneta e Sekiya, 2013; Hamstra-Wright, Bliven e Bay, 2014).

Aumento da ADM de flexão plantar foi considerado um fator de risco para SETM, pois com esse aumento, há também a maior probabilidade de os atletas aterrissarem com o antepé durante a corrida, isso pode causar um aumento na tensão na face anterior-medial da tíbia, se comparado com a aterrisagem usando o retropé. (Yates e White, 2004; Hamstra-Wright, Bliven e Bay, 2014). 
A força muscular é um importante fator a ser avaliado, visto que, atletas com fraqueza muscular do tríceps sural estão mais propensos a fadiga muscular, levando a alterações na biomecânica da corrida e aumento da tensão na tíbia. Também é importante ser avaliado a musculatura do core, do quadríceps e glúteo máximo, pois a fraqueza desses músculos centrais e estabilizadores é um fator de risco importante para lesões de membros inferiores. A força muscular de quadril e pelve é uma peça importante para manter o equilíbrio de forças entre a parte central do nosso corpo e as extremidades inferiores (Nascimento, 2020; Sievers e Busch, 2021).

Alterações biomecânicas ao longo da cadeia cinética também estão associadas a anormalidades estruturais em outras partes dos membros inferiores, podendo inclusive ter sua principal causa longe do local exato da lesão, como exemplo podemos citar uma alteração biomecânica em nível de coluna vertebral, articulação sacrilíaca ou até mesmo assimetrias na pelve podem contribuir para lesões de extremidades inferiores, dentre elas a SETM. (Yates e White, 2004; Nascimento, 2020).

\subsection{Diagnostico clinico}

Para realizar o diagnóstico da SETM é utilizado uma história clinica padronizada, seguido de um exame físico. Edwards, Wright e Hartman (2005) propôs uma série de etapas para o correto diagnóstico, bem como diferenciar a SETM de outras situações parecidas. Primeiramente deveria ser realizado a coleta de dados históricos, compreendendo perguntas sobre o início e localização da dor; em seguida, se a dor surgia devido a pratica de exercício físico e o que causava o alivio da dor; também deveria ser questionado se havia dores paralelas em áreas adjacentes; e por fim deveria ser questionado sobre a característica da dor sentida (dor pontuada, queimação, câimbra ou dormência). Caso a história clinica fosse compatível com a SETM, deveria ser realizado o exame físico, que consistia na palpação da borda póstero-medial e para verificar se haveria a presença de dor à palpação. Caso não houvesse dor ou se a dor apresentasse um tamanho menor que $5 \mathrm{~cm}$, deveria ser considerado outras lesões. A figura 2 apresenta um fluxograma baseado no estudo citado acima.

Yates e White (2004) nos disse que a SETM é considerada uma lesão por sobrecarga óssea, porem Beck e Osternig, (1994) e alguns outros estudos, sugerem que sua causa principal está mais relacionada com a periostite de tração. Isso significa que sua real causa ainda é desconhecida e aparentemente multifatorial. Franklyn e Oakes (2015) realizaram um estudo avaliando a precisão da ressonância magnética e tomografia computadorizada no diagnóstico da SETM. Porem com o decorrer do tempo e novos estudos foram realizados, firmou-se um consenso de que o exame clinico é o padrão ouro para diagnosticar a lesão, fazendo com que os exames de imagem sejam necessários apenas em alguns casos específicos, como por exemplo a especificidade de lesões em pacientes assintomáticos, diferenciação de lesões parecidas (fratura por estresse) e identificação de patologias raras (osteossarcoma), ou seja, quando há dúvida na fonte dos sintomas. (Wilder e Seth, 2004) 
Figura 2. Modelo adaptado do exame histórico e físico proposto por Edwards, Wright e Hartman (2005) para auxilio do diagnóstico clínico da SETM e diferenciação de demais lesões comuns a região distal da perna.

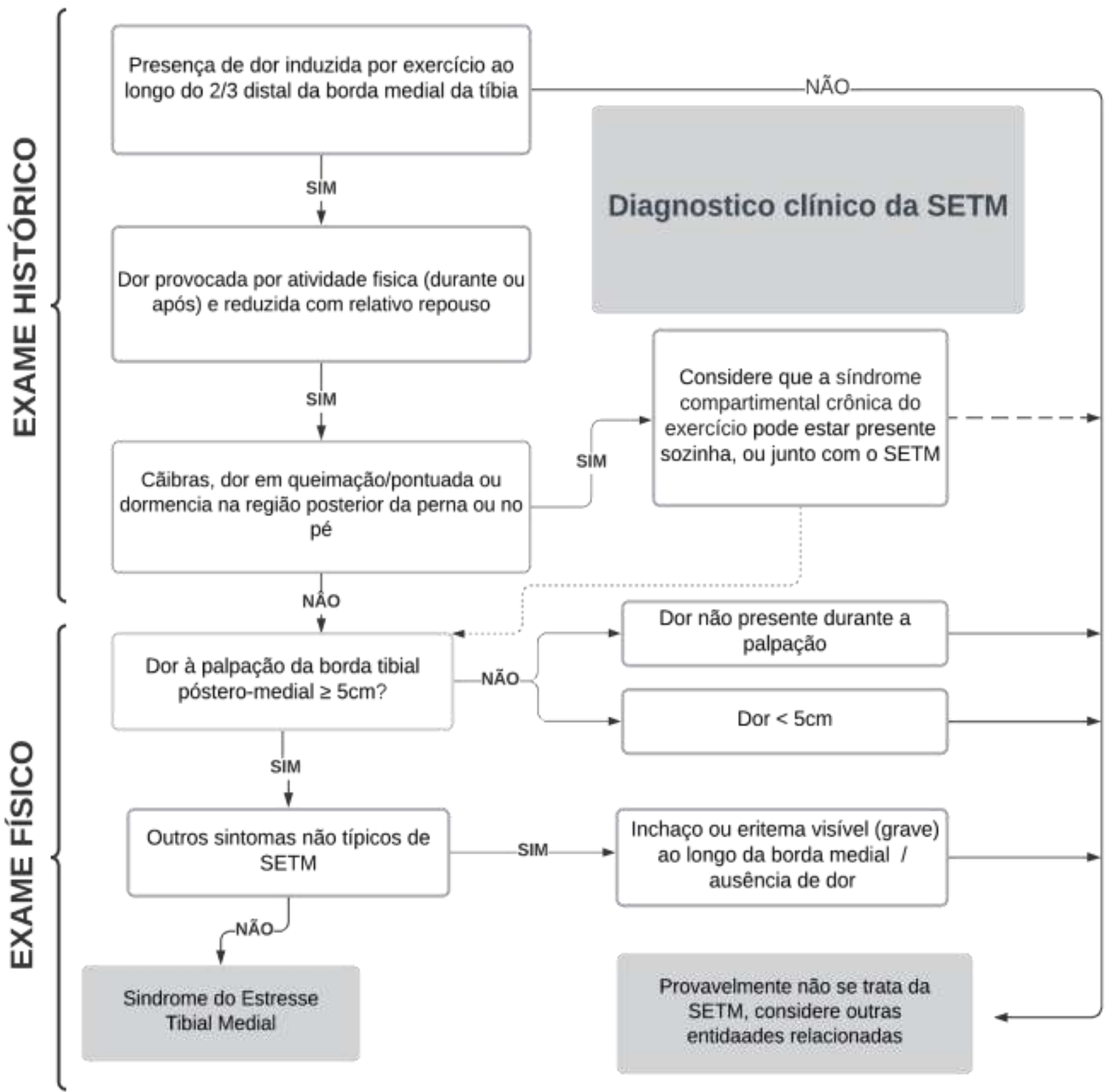

Fonte: https://pubmed.ncbi.nlm.nih.gov/16061959/_[adaptado]

\subsection{Alternativas conservadoras para a prevenção e tratamento da SETM.}

De acordo com Winters, et al. (2013) algumas alternativas conservadoras no tratamento estão disponíveis. Algumas demonstrando alguma relevância cientifica comprovada, como por exemplo: o tratamento utilizando laser de baixa potência, alongamento e fortalecimento da musculatura da panturrilha, uso de meias esportivas de compressão e órteses imobilizadoras. Há também algumas alternativas com pouquíssimos estudos que se mostraram potencialmente eficazes no tratamento da SETM, como por exemplo, a fonoforese, iontoforese, gelo-massagem e terapia extracorpórea por onda de choque. Porém, pela ausência de maiores estudos na área, nenhum dos tratamentos citados podem ser recomendados como protocolo padrão, pois os estudos apresentam níveis diferentes de viés em sua construção. 
Uma medida adotada para prevenção é a educação voltada a diminuição de impacto durante a corrida, diminuindo também a sobrecarga excessiva sobre a estrutura óssea da tíbia. essa medida é importante pois pequenos desgastes repetitivos se acumulam antes do surgimento dos sintomas (Winters et al., 2013). Uma grande parte das lesões frequentes aos corredores de rua são influenciadas por erros no programa de treinamento, portanto, faz parte da prevenção a implementação de um programa de educação ao corredor, fornecendo orientações a respeito das lesões relacionadas a corrida; exercícios de aquecimento e alongamento global, com ênfase em membros inferiores; tipos de tênis mais adequados ao paciente; e programa de evolução gradual da corrida. Entretanto todas essas medidas preventivas apresentam um nível de evidencia limitado, e, portanto, com benefícios controversos. (Alfayez, Ahmed e Alomar, 2017).

\subsection{Tratamento}

A atualmente, em grande parte dos tratamentos é incentivado a pratica da aplicação de crioterapia e repouso na fase inicial da SETM com o objetivo de alívio da dor e o retorno da pratica de corrida sem dor. Como foi dito por Alfayez, Ahmed e Alomar (2017), na fase aguda da lesão, a aplicação de gelo somado ao repouso é mostrado como o método mais eficaz de tratamento. Porém parar muitos atletas o repouso prolongado da atividade não é possível e outras alternativas de tratamento são necessárias para antecipar o retorno do atleta com segurança.

Durante a fase subaguda da lesão o objetivo do tratamento deve ser focado em modificar alguns aspectos da corrida que estejam contribuindo para o surgimento ou agravo da lesão. Uma mudança eficaz pode ser realizada no próprio programa de treinamento do atleta, diminuindo a distância percorrida semanalmente, bem como a frequência e intensidade em aproximadamente 50\% (Afonso, et al., 2020); também deve se levar em consideração a superfície em que se pratica a atividade, evitando a pratica da corrida em colinas, desníveis ou superfícies muito irregulares, onde a absorção de impacto se torna prejudicada. (Nero e Carvalho, 2019).

Segundo Brewer e Gregory, (2012) o alongamento do tendão do calcâneo e fortalecimento da panturrilha, sobretudo do musculo sóleo, tem sido implementado no tratamento de pacientes com SETM, pois um dos fatores predisponentes a SETM é o desequilíbrio de força dos músculos inversores e eversores do tornozelo. Portanto deve ser realizado exercícios de fortalecimento de tibial posterior, pois de acordo com Ross et al., (2018), o fortalecimento do tibial posterior pode promover uma correção de tornozelo em valgo e ainda diminuir a queda longitudinal do arco plantar, além de ajudar na diminuição de dor nas estruturas envolvidas. Os exercícios de fortalecimento de tibial posterior devem iniciar com graus de dificuldades baixos, com ênfase na parte concêntrica e ir evoluindo com maior contração excêntrica, estando alerta para evitar que haja estresse sobre as estruturas.

Segundo Hamstra-Wright, Bliven e Bay, (2014) o fortalecimento da musculatura do pé, auxiliam no aumento da rigidez do arco plantar, influenciando diretamente na diminuição da queda do navicular e na rotação medial da tíbia, e, portanto, sendo eficaz no tratamento.

O treinamento de equilíbrio e propriocepção é de fundamental importância para a educação neuromuscular. Com a propriocepção melhorada, há também o aumento da eficiência dos músculos estabilizadores das articulações, e essa eficiência ajuda o corpo a se manter estável em situações de desequilíbrio causado pelo terreno em que se está correndo. (Dugan e Weber 2007; Galbraith e Lavallee, 2009; Yamasaki, 2019).

Também é importante que seja feito um trabalho de fortalecimento do core, musculatura da região do abdômen, glúteos e quadril, pois são grandes responsáveis por manter a estabilidade do corpo, melhorando assim a biomecânica durante a corrida. (Leetun et al., 2004; Walker, 2011).

O tipo de tênis é um outro aspecto que interfere de forma direta no surgimento da SETM. Devem ser escolhidos aquele que melhor se adapte a biomecânica de cada atleta. O ideal é que o tênis seja capaz de absorver a maior quantidade de impacto, utilizando seu solado e palmilhas. Devem ter uma base estável e há a recomendação de que haja o revezamento de tênis durante a corrida para não comprometer sua capacidade de absorção de impacto. Tênis com palmilhas que beneficiem a o controle da 
pronação são benéficas, sobretudo para atletas com queda do navicular aumentado (Brochado, 2016; Alfayez, Ahmed e Alomar, 2017).

O tempo de reabilitação depende de muitos fatores, porém, ao decorrer dos dias ou semanas, o paciente poderá progredir a duração, intensidade e frequência do treinamento. Com a evolução, poderá acrescentar movimentos que ajudem a ter um melhor desempenho durante a corrida, como por exemplo, exercícios de salto e corrida em subida e descida, contanto que o paciente não apresente piora no sintoma de dor. (Yamasaki, 2019; Galbraith e Lavallee, 2009).

\section{Conclusão}

A SETM é uma lesão frequente em diversas modalidades que envolvam corrida, sua causa exata ainda é desconhecida, e tudo indica que seja causada por uma somatória de diversos fatores de risco intrínsecos e extrínsecos. Dentre os vários fatores, podemos destacar o índice de massa corpórea aumentado, queda do arco plantar (navicular drop test positivo), aumento da ADM da flexão plantar, diminuição de força muscular de MMII e alterações biomecânicas ao longo da cadeia cinética.

Algumas sugestões de prevenção foram identificadas na literatura, dentre elas, a implementação de um programa de orientações com o objetivo de executar a corrida com o mínimo de impacto possível e implementar um programa de corrida com evolução gradual, respeitando o limite do indivíduo. Quanto ao tratamento foi sugerido exercícios de alongamento do tendão do calcâneo e fortalecimento de panturrilha, e glúteos; exercícios proprioceptivos; fortalecimento da musculatura do core, afim de melhorar o controle do tronco durante a corrida.

Entretanto, apesar da grande incidência da SETM não foi identificado durante a revisão da literatura deste estudo, um protocolo padrão, considerado eficaz no tratamento da lesão, em decorrência disso, seu tratamento se concentra na apresentação clínica do paciente, sendo realizada intervenções generalistas. Portanto, é necessário a realização de mais estudos de boa qualidade na área, para que seja possível verificar a eficácia de intervenções fisioterapêutica de forma isolada no tratamento especifico da SETM.

\section{Referencias}

Afonso, M. dos S., Sousa, W. W. da S. e, Afonso, M. dos S., Junior, A. de O. S., Neves, F. B., \& Lourenção, L. G. (2020). Analysis of Injuries in Athletes Practitioners Amateur Race Street. Research, Society and Development, 9(3), e101932614.

Alfayez, S. M., Ahmed, M. L., Alomar, A. Z. (2017). A review article of medial tibial stress syndrome. J Musculoskelet Surg Res 2017;1:2-5

Beck, B. R.; Osternig, L. R. (1994). Medial tibial stress syndrome. The location of muscles in the leg in relation to symptoms., The Journal of Bone \& Joint Surgery: Jul 1994 - Volume 76 - Issue 7 - p 1057-1061

Belinda L., Lucy C.\& Angela, E. (2004) The Effect of Low-Dye Taping on Plantar Pressures, During Gait, in Subjects With Navicular Drop Exceeding 10 mm. Journal of Orthopaedic \& Sports Physical Therapy 2004 34:4, 201-209

Brewer, R. B., Gregory, A. J. (2012) Chronic lower leg pain in athletes: A guide for the differential diagnosis, evaluation, and treatment. Sports Health 2012;4:121-7.

Brochado, J. (2016). Análise biomecânica da influência do calçado desportivo na corrida.

Dugan S, Weber K. (2007). Stress fracture and rehabilitation. Phys Med Rehabil Clin N Am. 2007;18(3):401-16.

Edwards, P. H., Wright, M. L., \& Hartman, J. F. (2005). A Practical Approach for the Differential Diagnosis of Chronic Leg Pain in the Athlete. The American Journal of Sports Medicine, 33(8), 1241-1249.

Franklyn M, Oakes B. (2015). Aetiology and mechanisms of injury in medial tibial stress syndrome: Current and future developments. World J Orthop 2015; 6(8): 577-589

Fukuchi, R. K., \& Duarte, M. (2008). Análise cinemática comparativa da fase de apoio da corrida em adultos e idosos. Fisioterapia e Pesquisa, 15(1), 40-46.

Galbraith, R. M., \& Lavallee, M. E. (2009). Medial tibial stress syndrome: conservative treatment options. Current reviews in musculoskeletal medicine, 2(3), $127-133$.

Hamstra-Wright, K. L., Bliven, K. C., \& Bay, C. (2015). Risk factors for medial tibial stress syndrome in physically active individuals such as runners and military personnel: a systematic review and meta-analysis. British journal of sports medicine, 49(6), 362-369. 
Leetun, D. T., Ireland, M. L., Willson, J. D., Ballantyne, B. T., \& Davis, I. M. (2004). Core stability measures as risk factors for lower extremity injury in athletes. Medicine and science in sports and exercise, 36(6), 926-934.

Manoel, F. de A., Kravchychyn, A. C. P., Alves, J. C. C., \& Machado, F. A. (2015). Influência do nível de performance na estratégia de ritmo de corrida em prova de $10 \mathrm{~km}$ de corredores recreacionais. Revista Brasileira de Educação Física e Esporte, 29(3), 355-360.

Mazucato, T. (2018). Metodologia da pesquisa e do trabalho científico. la. ed. Penápolis: UNEPE.

Nascimento, G. S. (2020). Análise de lesões de membros inferiores recorrentes no curso básico da AMAN e sua relação com o treinamento físico militar.

Nero, D. da S. M. Carvalho, A. N. (2019). Aptidão física e saúde: exercício físico, saúde e fatores associados a lesões. volume 3. Pimenta Cultural, [S.L.], v. 3, n. 8, p. 77-97, 31 out. 2019. Pimenta Cultural.

Ross, M., Smith, M. D., Mellor, R., \& Vicenzino, B. (2018). Exercise for posterior tibial tendon dysfunction: a systematic review of randomised clinical trials and clinical guidelines. BMJ Open Sport — Exercise Medicine, 4.

Sabino, G. S., Rocha, I. C., Guimarães, C. Q., Alcântara, M. A. D., \& Felício, D. C. (2012). Análise da confiabilidade do teste clínico de queda do navicular. Fisioterapia em Movimento, 25(2), 301-309.

Sievers, M. E., \& Busch, A. M. (2021). Medial tibial stress syndrome: The relationship between gender and lower-extremity functional performance among collegiate track and field athletes. International Journal of Sports Medicine and Rehabilitation, 4(1), 16-16.

Tohmatsu, D. T. (2011). Muito além do futebol. Estudos sobre esportes no Brasil.

Viana, V. A. S. (2020). Síndrome do estresse tibial medial em militares.

Walker, B. (2011). Lesões no Esporte: uma Abordagem Anatômica.

Warden, S. J., Davis, I. S., \& Fredericson, M. (2014). Management and prevention of bone stress injuries in long-distance runners. The Journal of orthopaedic and sports physical therapy, 44(10), 749-765.

Wilder R, Seth S. (2004). Overuse injuries: tendinopathies, stress fractures, compartment syndrome, and shin splints. Clin Sports Med. 2004;23:55-81

Winters, M., Eskes, M., Weir, A., Moen, M. H., Backx, F. J., \& Bakker, E. W. (2013). Treatment of medial tibial stress syndrome: a systematic review. Sports medicine (Auckland, N.Z.), 43(12), 1315-1333

Yagi, S., Muneta, T., \& Sekiya, I. (2013). Incidence and risk factors for medial tibial stress syndrome and tibial stress fracture in high school runners. Knee Surgery, Sports Traumatology, Arthroscopy, 21(3), 556-563.

Yamasaki, S. (2019). A Review of the Treatment and Prevention Options for Medial Tibial Stress Syndrome.

Yamato, T. P., Saragiotto, B. T., \& Lopes, A. D. (2011). Prevalência de dor musculoesquelética em corredores de rua no momento em que precede o início da corrida. Revista Brasileira de Ciências do Esporte, 33(2), 475-482

Yates, B., \& White, S. (2004). The incidence and risk factors in the development of medial tibial stress syndrome among naval recruits. The American journal of sports medicine, 32(3), 772-780. 